\title{
A Modeling Study of the Initial Formation of Polar Lows in the Vicinity of the Arctic Front
}

\author{
Igor Mingalev, Konstantin Orlov, and Victor Mingalev \\ Polar Geophysical Institute, Kola Scientific Center of the Russian Academy of Sciences, Apatity, Murmansk Region 184209, Russia \\ Correspondence should be addressed to Victor Mingalev; mingalev@pgia.ru
}

Received 19 April 2013; Accepted 9 December 2013; Published 27 February 2014

Academic Editor: P. C. S. Devara

Copyright (C) 2014 Igor Mingalev et al. This is an open access article distributed under the Creative Commons Attribution License, which permits unrestricted use, distribution, and reproduction in any medium, provided the original work is properly cited.

\begin{abstract}
A regional mathematical model of the wind system of the lower atmosphere, developed recently in the Polar Geophysical Institute, is applied to investigate the initial stage of the formation of polar lows at latitudes of the European Arctic. The mathematical model is based on numerical solving of nonsimplified gas dynamic equations and produces three-dimensional distributions of the atmospheric parameters in the height range from 0 to $15 \mathrm{~km}$ over a limited region of the Earth's surface. Simulation results indicated that the origin of a convexity in the configuration of the arctic front can lead to the formation of a polar low during the period of about one day.
\end{abstract}

\section{Introduction}

A polar low (PL) is an intense mesoscale atmospheric low pressure weather system (depression), involving strong winds, that originates over polar oceans in both the Northern and Southern hemispheres. Polar lows have been referred to by other terms, such as polar mesoscale cyclone (PMC), arctic hurricane, and cold air depression. Polar lows are characterized by small sizes and short lifetimes in comparison with tropical cyclones. Nevertheless, polar lows can cause wave surges, threat to ships, coastal flooding, and numerous fatalities for coastal communities. Therefore, prediction of polar low formation is a very important problem.

During the last five decades, polar lows have been investigated both experimentally (see [1-9] and references therein) and theoretically (see [10-16]). Also, to investigate the dynamics of polar lows mathematical models have been applied (e.g., see [17-22]).

Not long ago, in the Polar Geophysical Institute (PGI), two nonhydrostatic mathematical models of the wind system in the Earth's atmosphere have been developed. The first model enables to calculate three-dimensional global distributions of the zonal, meridional, and vertical components of the wind at levels of the troposphere, stratosphere, mesosphere, and lower thermosphere $[23,24]$. The characteristic feature of this model is that it does not include the internal energy equation for the atmospheric gas. Instead, the global temperature field is assumed to be a given distribution obtained from the NRLMSISE-00 empirical model [25]. This mathematical model has been applied in order to investigate numerically how the horizontal nonuniformity of the atmospheric gas temperature affects the formation of the middle atmosphere global circulation for conditions corresponding to different seasons $[26,27]$ and how solar activity affects the formation of the large-scale global circulation of the mesosphere and lower thermosphere [28].

The second mathematical model, developed not long ago in the PGI, is a limited area mathematical model of the wind system of the lower atmosphere, which produces threedimensional distributions of the atmospheric parameters in the height range from 0 to $15 \mathrm{~km}$ over a limited region of the Earth's surface, with the internal energy equation for the air being included in the model $[29,30]$. This regional model has been utilized in order to investigate numerically the mechanisms responsible for the formation of large-scale vortices over the ocean surface at tropical latitudes [3133]. In these investigations, it was shown that the origin of convexities in the form of the intertropical convergence zone (ITCZ), having distinct configurations, can lead to the formation of different large-scale vortices, in particular, a 
cyclonic vortex, pair of cyclonic-anticyclonic vortices, and pair of cyclonic vortices, during the period not longer than three days.

Also, the latter regional mathematical model has been applied to verify the hypothesis of the influence of the shape of the arctic front on the initial formation of polar lows. This hypothesis has been advanced and confirmed in the studies by Mingalev et al. $[34,35]$. In these studies, simulations were performed for two cases when, at the initial moment, the simulation domain is intersected by the arctic front with different configurations. It was shown that the origin of a convexity in the configuration of the arctic front can lead to the formation of a polar low during the period of about one day.

The purpose of the present work is to continue the investigation of the initial stage of the formation of polar lows at latitudes of the European Arctic, applying the regional mathematical model of the wind system of the lower atmosphere, developed in the PGI. Time-dependent modeling is performed for various cases in which the initial forms of the arctic front are different and contain convexities with distinct shapes, with the distributions of wind velocity in the vicinity of the arctic front being chosen different.

\section{Mathematical Model}

The limited area nonhydrostatic mathematical model of the wind system of the lower atmosphere, developed not long ago at the Polar Geophysical Institute, is utilized in the present study. In this model, the atmospheric gas is considered as a mixture of air and water vapor, in which two types of precipitating water (namely, water microdrops and ice microparticles) can exist. The model is based on the numerical solution of the system of transport equations containing the equations of continuity for air and for the total water content in all phase states, momentum equations for the zonal, meridional, and vertical components of the air velocity, and energy equation. The peculiarity of the model is that the vertical component of the air velocity is calculated without using the hydrostatic equation. Instead, the vertical component of the air velocity is obtained by means of a numerical solution of the appropriate momentum equation, with whatever simplifications of this equation being absent. In the momentum equations for all components of the air velocity, the effect of the turbulence on the mean flow is taken into account by using an empirical subgrid-scale parameterization similarly to the global circulation model of the Earth's atmosphere developed earlier in the PGI $[23,24]$.

In general, the applied mathematical model is based on numerical solving of nonsimplified gas dynamic equations and produces three-dimensional time-dependent distributions of the wind components, temperature, air density, water vapor density, concentration of microdrops of water, and concentration of ice particles. The model takes into account heating/cooling of the air due to absorption/emission of infrared radiation, as well as due to phase transitions of water vapor to microdrops of water and ice particles, which play an important role in energetic balance. The finite-difference method and explicit scheme are applied for solving the system of governing equations.

The following variables are computed in the model calculations at each grid node: the temperature of the mixture of air and water vapor, $T$; densities of air and water vapor, $\rho_{a}$ and $\rho_{v}$, respectively; hydrodynamic velocity of the mixture (a $3 \mathrm{D}$ vector), $\vec{v}$; and the total mass of water microdrops and ice microparticles in a unit volume, $\rho_{w}$ and $\rho_{i}$, respectively. The governing equations in vectorial form can be written as follows:

$$
\begin{gathered}
\frac{\partial \rho_{a}}{\partial t}+\operatorname{div}\left(\rho_{a} \vec{v}\right)=0, \\
\frac{\partial\left(\rho_{v}+\rho_{w}+\rho_{i}\right)}{\partial t}+\operatorname{div}\left[\rho_{v} \vec{v}+\rho_{w}\left(\vec{v}+\vec{v}_{w}^{\text {prec }}\right)\right. \\
\left.+\rho_{i}\left(\vec{v}+\vec{v}_{i}^{\text {prec }}\right)\right]=0, \\
\frac{\partial\left(\rho_{\text {mix }} \vec{v}\right)}{\partial t}+\operatorname{div}\left(\rho_{\text {mix }} \vec{v} \otimes \vec{v}\right) \\
\frac{\partial W}{\partial t}+\operatorname{div}\left[W_{\text {mix }} \vec{v}+W_{w}\left(\vec{v}+\vec{v}_{w}^{\text {prec }}\right)+W_{i}\left(\vec{v}+\vec{v}_{i}^{\text {prec }}\right)\right] \\
=\left[\rho_{\text {mix }} \vec{v}+\rho_{w}\left(\vec{v}+\vec{v}_{w}^{\text {prec }}\right)+\rho_{i}\left(\vec{v}+\vec{v}_{i}^{\text {prec }}\right), \vec{F}\right] \\
+\operatorname{div}(\hat{\tau} \cdot \vec{v}-p \vec{v}-\vec{j})+Q,
\end{gathered}
$$

where $\vec{v}_{w}^{\text {prec }}$ and $\vec{v}_{i}^{\text {prec }}$ are the precipitation velocities of water microdrops and ice microparticles, respectively, conditioned by the presence of an external force field and determined from the Stokes relation with Cunningham's correction; $\rho_{\text {mix }}=$ $\rho_{a}+\rho_{v} ; p$ is the pressure of the mixture defined as $p=\left(\rho_{a} R_{a}+\right.$ $\left.\rho_{v} R_{v}\right) T$, where $R_{a}$ and $R_{v}$ are the gas constants of air and water vapour, respectively; $\widehat{\tau}$ is the extra stress tensor whose components are given by the rheological equation of state or the law of viscous friction which is the same as in the global circulation model of the Earth's atmosphere developed earlier $[23,24]$, with the effect of a small-scale turbulence, having the scales equal and less than the steps of finite-difference approximations, on the mean flow having been taken into account; $\vec{F}$ is the acceleration due to an external force field, which consists of the gravity acceleration, Coriolis acceleration, and acceleration of translation, that can be written in the form

$$
\vec{F}=\vec{g}-2 \vec{\Omega} \times \vec{v}-\vec{\Omega} \times(\vec{\Omega} \times \vec{r})
$$

where $\vec{g}$ is the acceleration due to gravity, $\vec{\Omega}$ is the Earth's angular velocity, and $\vec{r}$ is a radius vector from the center of the Earth to the point where the equation is applied. The following notations are used in (4)

$$
\begin{gathered}
W_{i}=\rho_{i}\left[\frac{1}{2}\left(\vec{v}+\vec{v}_{i}^{\text {prec }}\right)^{2}+C_{i} T\right], \\
W_{w}=\rho_{w}\left[\frac{1}{2}\left(\vec{v}+\vec{v}_{w}^{\text {prec }}\right)^{2}+C_{w}\left(T-T_{0}\right)+q_{\text {mel }}+C_{i} T_{0}\right], \\
W=W_{\text {mix }}+W_{w}+W_{i},
\end{gathered}
$$


where $C_{i}$ and $C_{w}$ are the specific heat capacities of ice and water, respectively, which are assumed to be constant; $T_{0}$ is the freezing temperature of water; $q_{\mathrm{mel}}$ is the specific heat of ice melting at $T=T_{0} ; q_{\mathrm{ev}}^{0}$ is the specific heat of water evaporation at $T=T_{0}$; also, the vector of heat flux, $\vec{j}$, is given by the well-known formula, $\vec{j}=-\widehat{\lambda} \nabla T$, where $\widehat{\lambda}$ is the symmetric tensor of thermal conductivity coefficients; and $Q$ is the rate of change of energy in a unit volume due to absorption/emission of infrared radiation. Concrete expressions of the model parameters, those appearing in (1)(4), may be found in the studies of Belotserkovskii et al. $[29,30]$.

It should be emphasized that the model assumes that the water microdrops can exist only in the presence of saturated water vapor on condition that $T \geq T_{0}$, while the ice microparticles can exist only in the presence of saturated water vapor on condition that $T \leq T_{0}$. At $T=T_{0}$, the temperature of the matter cannot increase until all ice microparticles melt, and it cannot decrease until all water microdrops freeze.

It is supposed that the three-dimensional simulation domain of the model is a part of a spherical layer stretching from land and ocean surface up to the altitude of $15 \mathrm{~km}$ over a limited region of the Earth's surface. The dimensions of this region in longitudinal and latitudinal directions are $36^{\circ}$ and $25^{\circ}$, respectively. The finite-difference method and explicit scheme are applied for solving the system of governing equations. The calculated parameters are determined on a uniform grid. The latitude and longitude steps are equal to $0.08^{\circ}$, and height step is equal to $200 \mathrm{~m}$. Complete details of the utilized finite-difference method and numerical schemes have been presented in the paper of Mingalev et al. [36]. More complete details of the applied regional mathematical model may be found in the studies of Belotserkovskii et al. $[29,30]$ and Mingalev et al. [34].

\section{Presentation and Discussion of Results}

As was noted earlier, the application of the limited area mathematical model of the wind system of the lower atmosphere, described above, has allowed identifying a new mechanism of the initial formation of the tropical cyclones in the vicinity of the intertropical convergence zone [31-33]. In the mentioned works, it was shown that the origin of a convexity of the intertropical convergence zone, having the specific forms, can lead to the formation of not only a single cyclonic vortex but also a pair of large-scale vortices. The simulation results have shown that the key factor in the mechanism of the formation of tropical large-scale vortices is the origin of a convexity in the configuration of the intertropical convergence zone. The origin of the convexity can lead to a rise of instability of stream air flow present in the intertropical convergence zone. This instability leads to considerable transformation of the wind field and to formation of tropical large-scale vortices in the vicinity of the initial position of the intertropical convergence zone in the course of time.

The simulation results, described above, have prompted the authors of the present study to advance the hypothesis of the influence of the shape of the arctic front on the initial formation of polar lows [34, 35], in despite of considerable distinctions between air flows in the intertropical convergence zone and arctic front.

The arctic front separates the cold arctic air masses from warmer air masses. The arctic front can also be defined as the semipermanent, semicontinuous boundary of the cold arctic air mass. As a rule, the extension of the arctic front in the meridional direction does not exceed $200 \mathrm{~km}$ and its length in the zonal direction may be more than $2000 \mathrm{~km}$. The direction, along which the arctic front lies, deviates from the zonal direction for less than 20 degrees, as a rule. It is known from observations that, in an arctic front, a zonal flow of air is westward at more northern latitudes relatively to the centerline of the arctic front. On the contrary, a zonal flow of air is eastward at more southern latitudes relatively to the centerline of the arctic front. In an arctic front, a meridional wind velocity directs towards the centerline of an arctic front at levels less than approximately $2.5 \mathrm{~km}$ and directs from the centerline of an arctic front at levels higher than approximately $2.5 \mathrm{~km}$. A vertical wind velocity in an arctic front is upward. Therefore, an arctic front may be considered as a system consisting of two air streams moving in opposite directions in the ambient atmospheric gas, with strong velocity shear taking place close to the centerline of an arctic front.

In our calculations, we define the initial and boundary conditions as consistent with observational data and for the situations when the arctic front intersects the simulation domain in the west-east direction. Calculations are made for various cases in which the initial forms of the arctic front are different and contained convexities with distinct shapes. Moreover, simulations are performed for the cases in which the modules of the zonal wind velocities at more northern latitudes relatively to the centerline of the arctic front are less than those at more southern latitudes relatively to it. Since the obtained results are different, it is convenient to present them separately.

3.1. A Convexity in the South Direction. Initially, let us consider the first case when, at the initial moment, the arctic front contains a convexity in the south direction, with the deviation achieving a value of one hundred kilometers. The initial form of the arctic front may be easily seen from the panel (a) of Figure 1, where it is like a dark curved band between two light curved bands. It is essential to note that, in the considered first case, the fields of the module of the horizontal velocity are approximately symmetric relatively to the centerline of the arctic front not only inside it but also beyond the arctic front.

The time evolution of model parameters was numerically simulated using the mathematical model during the period of about two days. The results of time-dependent modeling are partly shown in Figure 1. The panel (a) of Figure 1 shows a distribution of the wind horizontal component at a height of $600 \mathrm{~m}$ at the initial moment. In the panels (b) and (c), the same distribution is shown for time moments 20 and $40 \mathrm{~h}$ after the simulation beginning, respectively. As can be seen from this figure, in the course of time, the initial distribution of horizontal component of the air velocity was considerably 


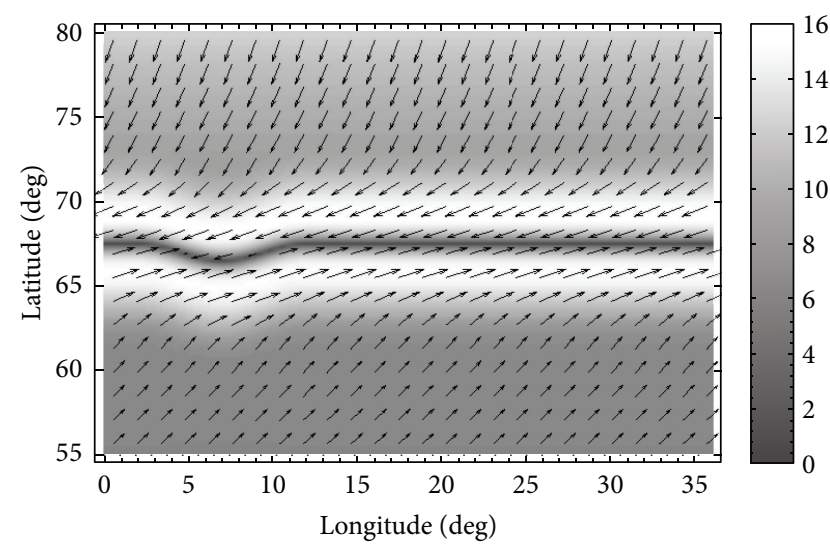

(a)

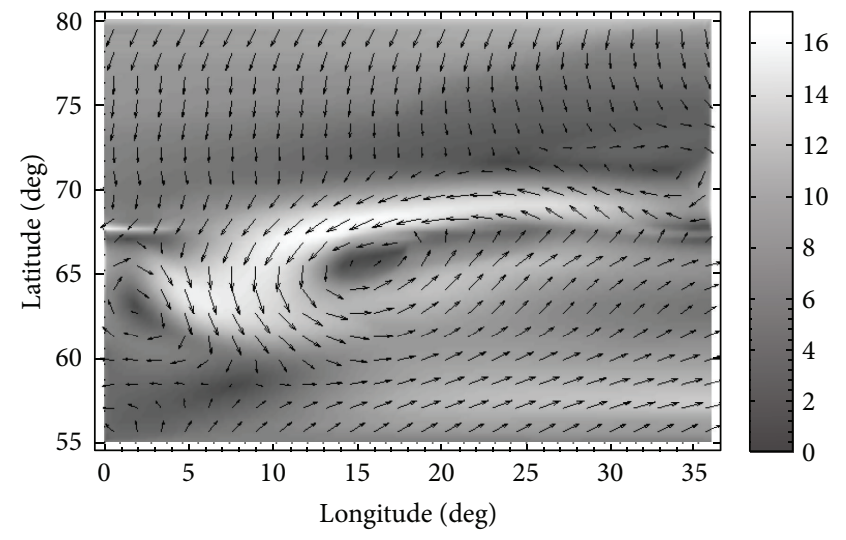

(b)

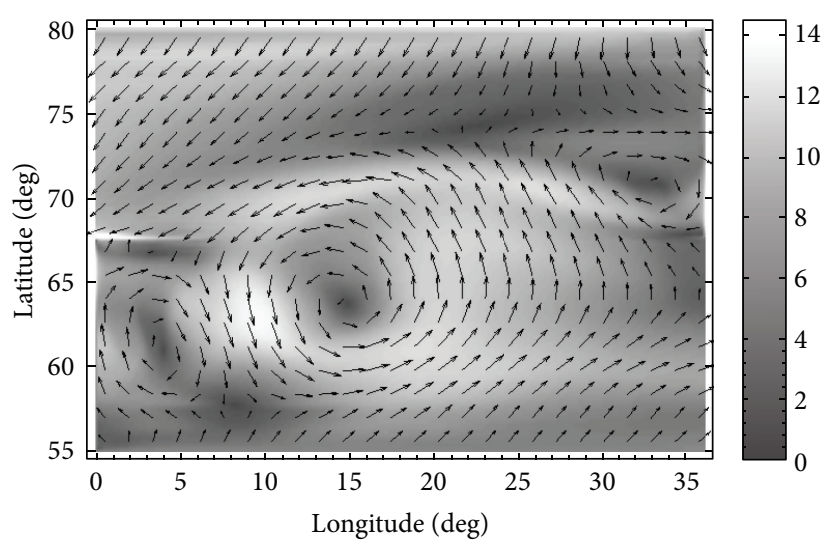

(c)

FIGURE 1: The distributions of horizontal component of the air velocity at the altitude of $600 \mathrm{~m}$, assigned at the initial moment (a), computed 20 hours after the beginning of calculations (b) and computed 40 hours after the beginning of calculations (c). The results are obtained for the first initial configuration of the arctic front, with the fields of the module of the horizontal velocity being approximately symmetric relatively to the centerline of the arctic front. The degree of shadowing of the figures indicates the module of the velocity in $\mathrm{m} / \mathrm{s}$.

transformed. The panel (a) of Figure 1 shows that at the initial moment, the center of the bent part of the front is located $7^{\circ}$ eastward from the western boundary of the simulation region. One can see in the panel (b) of Figure 1 that, $20 \mathrm{~h}$ after the simulation beginning, a destruction of the arctic front has happened in the place of the bend, and a cyclonic vortex with the center located at latitude of approximately $66^{\circ} \mathrm{N}$ and approximately $16^{\circ}$ eastward from the western boundary of the simulation region is formed. The wind velocity within this vortex reaches $17.5 \mathrm{~m} / \mathrm{s}$ and the horizontal dimension across this vortex is approximately $600-800 \mathrm{~km}$. One can see in the panel (c) of Figure 1 that $40 \mathrm{~h}$ after the simulation beginning the center of the cyclonic vortex is located at the latitude of approximately $64^{\circ} \mathrm{N}$ and approximately $15.5^{\circ}$ eastward from the western boundary of the simulation region. The wind velocity within the vortex reaches $14.5 \mathrm{~m} / \mathrm{s}$.

The simulation results presented in Figure 1 show that, at the presence at the initial moment of the part of the arctic front bent to the south, eastward from this part, approximately in $20 \mathrm{~h}$, a cyclonic vortex is formed which moves southward with a velocity of approximately $10 \mathrm{~km} / \mathrm{h}$. The maximum wind velocity within the vortex is reached approximately $20 \mathrm{~h}$ after the simulation beginning, and then it begins to decrease slowly.

In addition, we consider the second case when, at the initial moment, the fields of the module of the horizontal velocity are not symmetric relatively to the centerline of the arctic front not only inside it but also beyond the arctic front. The initial form of the arctic front may be easily seen from the panel (a) of Figure 2, where it is like a dark curved band between two light curved bands.

The time evolution of atmospheric parameters was numerically simulated using the mathematical model during the period of about two days. The results of time-dependent modeling are partly shown in Figure 2 . The panel (a) of Figure 2 shows a distribution of the horizontal wind component at a height of $600 \mathrm{~m}$ at the initial moment. In the panels (b) and (c), the same distribution is shown for time moments 20 and $40 \mathrm{~h}$ after the simulation beginning, respectively. It can be seen from this figure that, in the course of time, the initial distribution of horizontal component of the air velocity was considerably transformed. The panel (a) of Figure 2 shows 


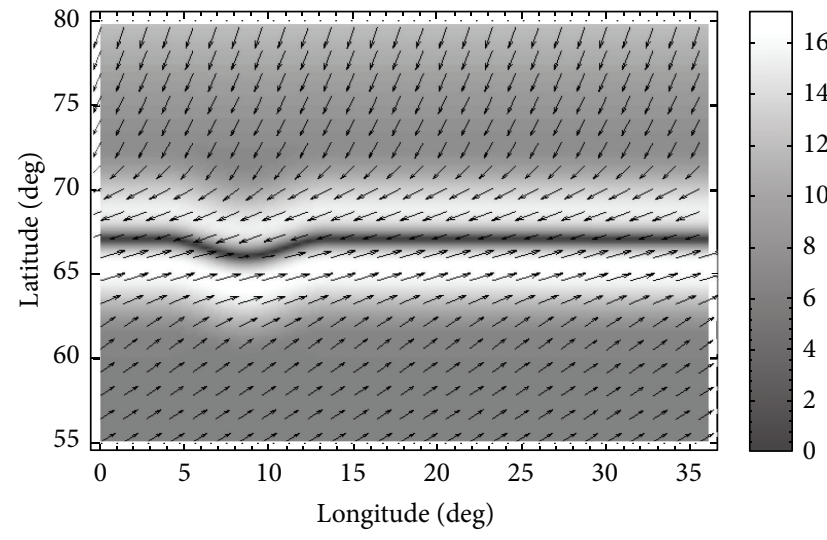

(a)

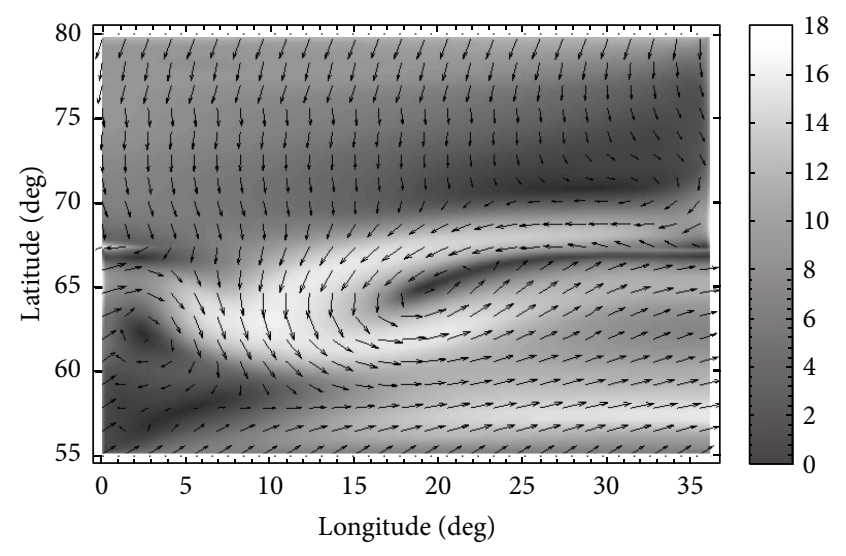

(b)

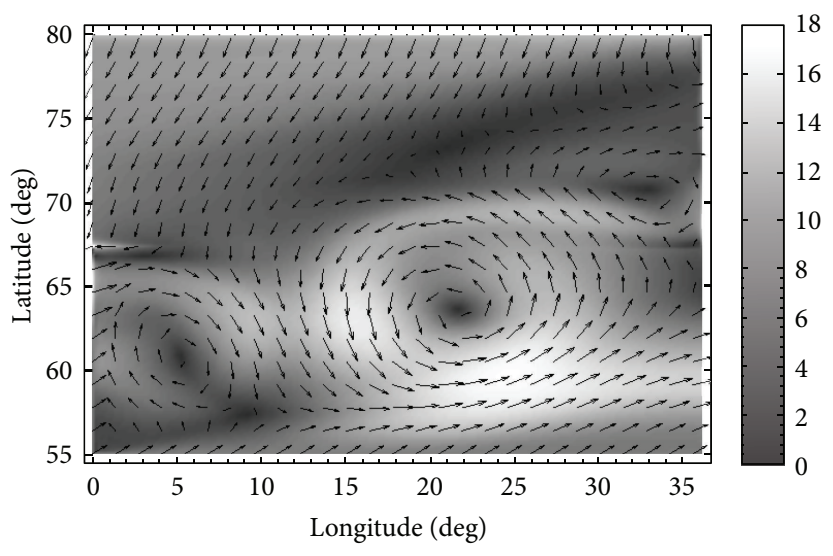

(c)

Figure 2: The same as in Figure 1, but obtained for the second case when, at the initial moment, the modules of the zonal wind velocities at more northern latitudes relatively to the centerline of the arctic front are less than those at more southern latitudes relatively to it. The degree of shadowing of the figures indicates the module of the velocity in $\mathrm{m} / \mathrm{s}$.

that, at the initial moment, the center of the bent part of the front is located $8^{\circ}$ eastward from the western boundary of the simulation region. One can see in the panel (b) of Figure 2 that $20 \mathrm{~h}$ after the simulation beginning a destruction of the arctic front has happened in the place of the bend, and a cyclonic vortex with the center located at latitude of approximately $65^{\circ} \mathrm{N}$ and approximately $18^{\circ}$ eastward from the western boundary of the simulation region is formed. The wind velocity within this vortex reaches $18 \mathrm{~m} / \mathrm{s}$ and the horizontal dimension across this vortex is approximately $700 \mathrm{~km}$. One can see in the panel (c) of Figure 2 that $40 \mathrm{~h}$ after the simulation beginning the center of the cyclonic vortex is located at the latitude of approximately $63^{\circ} \mathrm{N}$ and approximately $22^{\circ}$ eastward from the western boundary of the simulation region. The wind velocity within the vortex reaches $17 \mathrm{~m} / \mathrm{s}$.

The simulation results presented in Figure 2 show that, at the presence at the initial moment of the part of the arctic front bent to the south, when the fields of the module of the horizontal velocity are not symmetric relatively to the centerline of the arctic front, eastward from this part, approximately in $20 \mathrm{~h}$, a cyclonic vortex is formed which moves to the south and to the east with a velocity of approximately $11 \mathrm{~km} / \mathrm{h}$. The maximum wind velocity within the vortex is reached approximately $20 \mathrm{~h}$ after the simulation beginning, and then it begins to decrease slowly. The radius of this largescale cyclonic vortex is about $600-800 \mathrm{~km}$.

3.2. A Convexity in the North Direction. In the previous subsection, we have considered two cases of the initial configuration of the arctic front containing the convexity in the south direction. In the present subsection, we consider cases when the initial configuration of the arctic front contains the convexity in the north direction.

Initially, let us consider the third case when, at the initial moment, the arctic front contains a convexity in the north direction, with the fields of the module of the horizontal velocity being approximately symmetric relatively to the centerline of the arctic front not only inside it but also beyond the arctic front. The initial form of the arctic front may be 


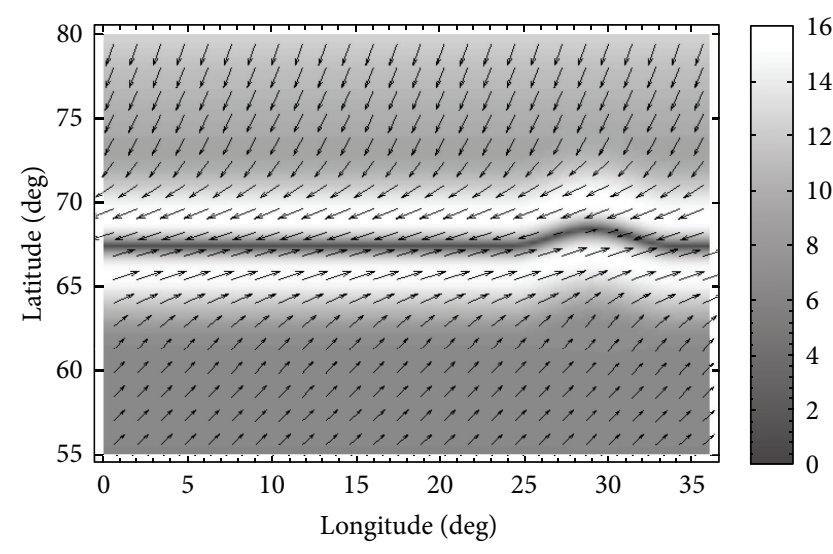

(a)

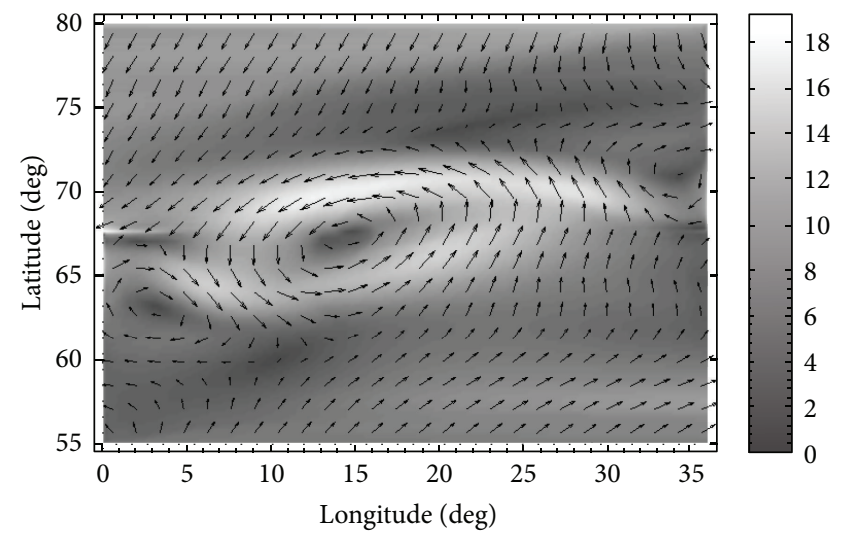

(b)

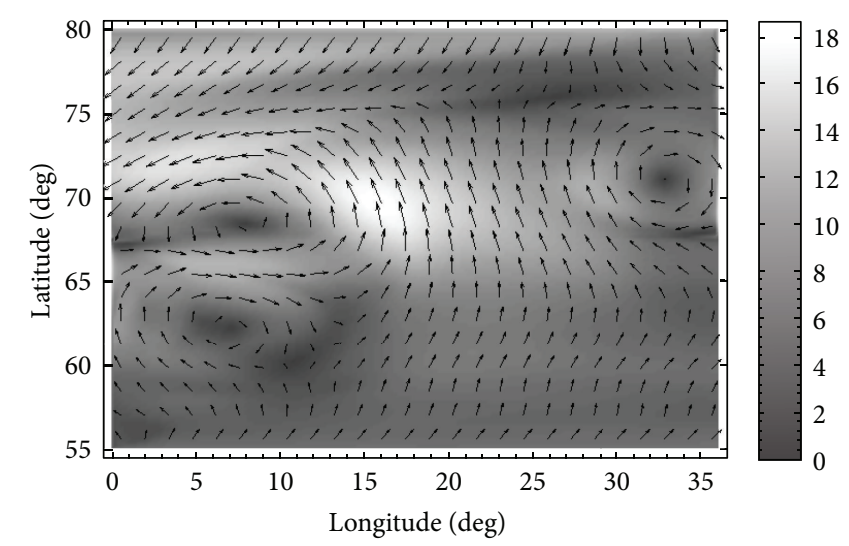

(c)

FIGURE 3: The distributions of horizontal component of the air velocity at the altitude of $600 \mathrm{~m}$, assigned at the initial moment (a), computed 20 hours after the beginning of calculations (b) and computed 40 hours after the beginning of calculations (c). The results are obtained for the third initial configuration of the arctic front, with the fields of the module of the horizontal velocity being approximately symmetric relatively to the centerline of the arctic front. The degree of shadowing of the figures indicates the module of the velocity in $\mathrm{m} / \mathrm{s}$.

easily seen from the panel (a) of Figure 3, where it is like a dark curved band between two light curved bands. The center of the bent part of the front is $29^{\circ}$ eastward from the western boundary of the simulation region.

The time evolution of model parameters was numerically simulated using the mathematical model during the period of about 1.5 days. The results of time-dependent modeling are partly shown in Figure 3. One can see in the panel (b) of Figure 3 that, $20 \mathrm{~h}$ after the simulation beginning, a cyclonic vortex with the center located at latitude of approximately $67^{\circ} \mathrm{N}$ and approximately $15^{\circ}$ eastward from the western boundary of the simulation region is formed. One can see that the wind velocity within this vortex reaches $18 \mathrm{~m} / \mathrm{s}$ in its northern part and the horizontal dimension of this vortex is approximately $600-800 \mathrm{~km}$. The panel (c) of Figure 3 shows that, $40 \mathrm{~h}$ after the simulation beginning, the cyclonic vortex center lies at latitude of approximately $68^{\circ} \mathrm{N}$ and approximately $8.5^{\circ}$ eastward from the western boundary of the simulation region. The wind velocity in the vortex reaches $18 \mathrm{~m} / \mathrm{s}$.

Thus, the simulation results presented in Figure 3 show that, at the presence at the initial moment of a part of the arctic front bent to the north, eastward from this part approximately during $15 \mathrm{~h}$, a cyclonic vortex is formed which moves westward with a velocity of $10-15 \mathrm{~km} / \mathrm{h}$. The maximum wind velocity in the vortex is reached within the time interval 15-20 $\mathrm{h}$ after starting the simulation, and then it begins to decrease slowly.

In addition, we consider the fourth case when, at the initial moment, the fields of the module of the horizontal velocity are not symmetric relatively to the centerline of the arctic front not only inside it but also beyond the arctic front. The initial form of the arctic front may be easily seen from the panel (a) of Figure 4, where it is like a dark curved band between two light curved bands. The center of the bent part of the front is $27^{\circ}$ eastward from the western boundary of the simulation region.

The time evolution of atmospheric parameters was numerically simulated using the mathematical model during the period of about two days. The results of time-dependent modeling are partly shown in Figure 4 . It can be seen from this figure that, in the course of time, the initial distribution of horizontal component of the air velocity was considerably transformed. One can see in the panel (b) of Figure 4 that, 


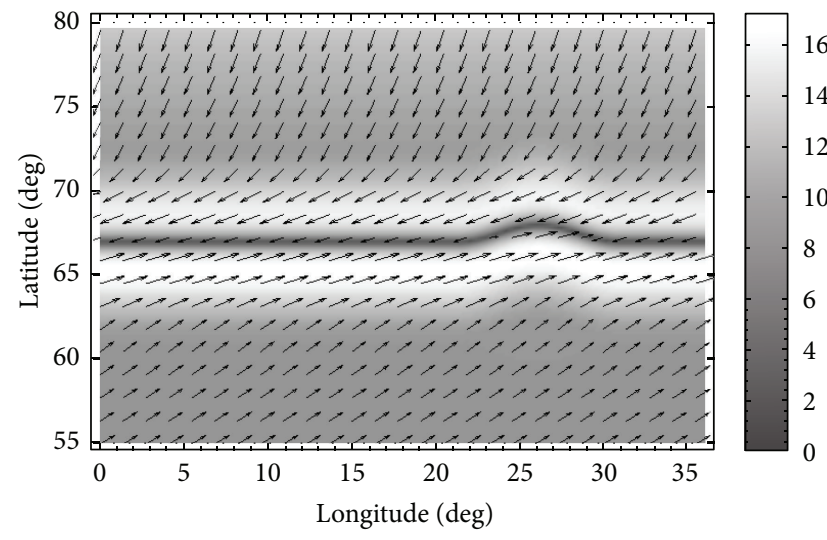

(a)

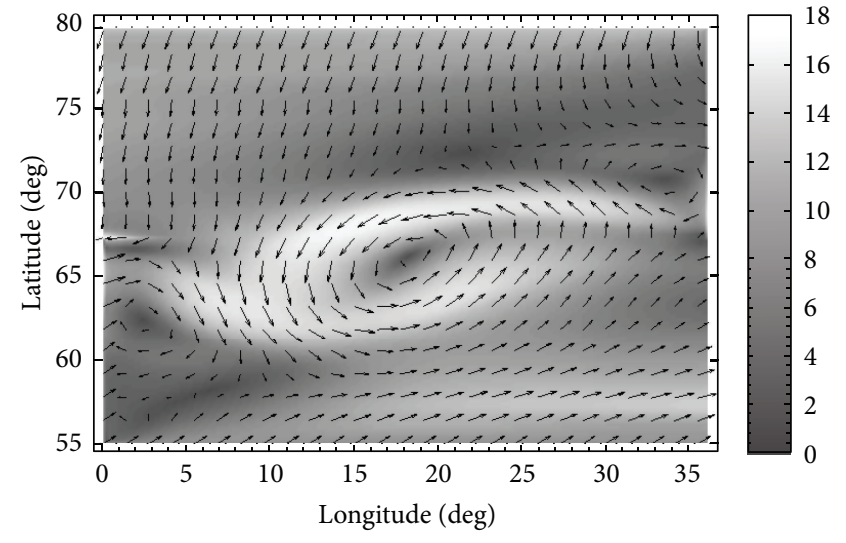

(b)

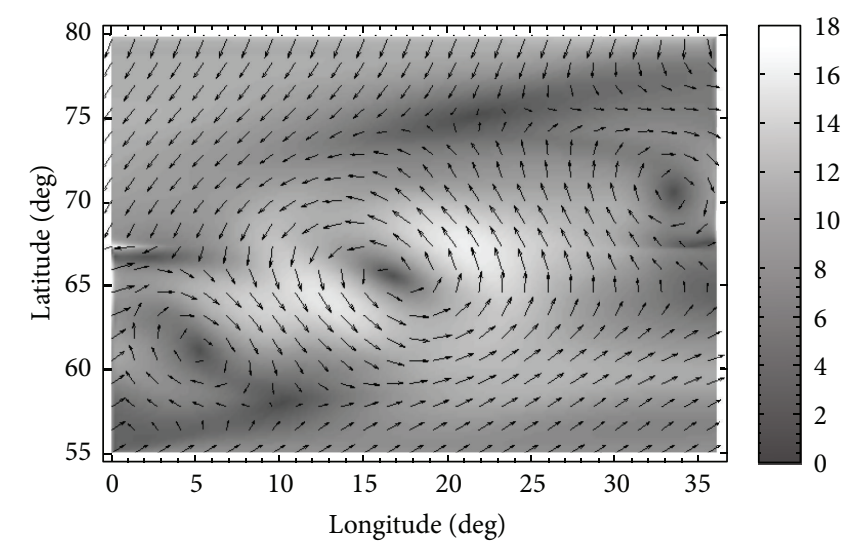

(c)

Figure 4: The same as in Figure 3, but obtained for the fourth case when, at the initial moment, the modules of the zonal wind velocities at more northern latitudes relatively to the centerline of the arctic front are less than those at more southern latitudes relatively to it. The degree of shadowing of the figures indicates the module of the velocity in $\mathrm{m} / \mathrm{s}$.

$20 \mathrm{~h}$ after the simulation beginning, a cyclonic vortex with the center located at latitude of approximately $67^{\circ} \mathrm{N}$ and approximately $18^{\circ}$ eastward from the western boundary of the simulation region is formed. One can see that the wind velocity within this vortex reaches $18 \mathrm{~m} / \mathrm{s}$ in its northern part and the horizontal dimension of this vortex is approximately $600-800 \mathrm{~km}$. The panel (c) of Figure 4 shows that, $40 \mathrm{~h}$ after the simulation beginning, the cyclonic vortex center lies at latitude of approximately $65^{\circ} \mathrm{N}$ and approximately $17^{\circ}$ eastward from the western boundary of the simulation region. The wind velocity in the vortex reaches $18 \mathrm{~m} / \mathrm{s}$.

The simulation results presented in Figure 4 show that, at the presence at the initial moment of the part of the arctic front bent to the north, when the fields of the module of the horizontal velocity are not symmetric relatively to the centerline of the arctic front, westward from this part, approximately in $20 \mathrm{~h}$, a cyclonic vortex is formed which moves to the south and to the west with a velocity of approximately $5-10 \mathrm{~km} / \mathrm{h}$. The maximum wind velocity within the vortex is reached approximately $20 \mathrm{~h}$ after the simulation beginning, and then it begins to decrease slowly. The radius of this largescale cyclonic vortex is about $600-800 \mathrm{~km}$.

Thus, the simulation results show that the origin of a convexity in the configuration of the arctic front can lead to the formation of a polar low during the period for about one day, with the rotational center of the polar low being close to the southern edge of the initial position of the arctic front.

Let us compare the simulation results with experimental data. The Space Monitoring Information Support laboratory of the Space Research Institute of the Russian Academy of Sciences has collected the Regional Data Archive, obtained from the spacecraft mission NOAA (http://smis.iki.rssi.ru). As examples of data from this Regional Data Archive, fragments of cloud cover distributions over the part of Norwegian Sea are presented in Figures 5 and 6.

From Figure 5 one can see that the cloud cover distribution has the convexity in the north direction that may be connected with the configuration of the arctic front, very likely. From Figure 6 one can see that, after approximately 23 hours, a cyclonic vortex with the center located at latitude of 


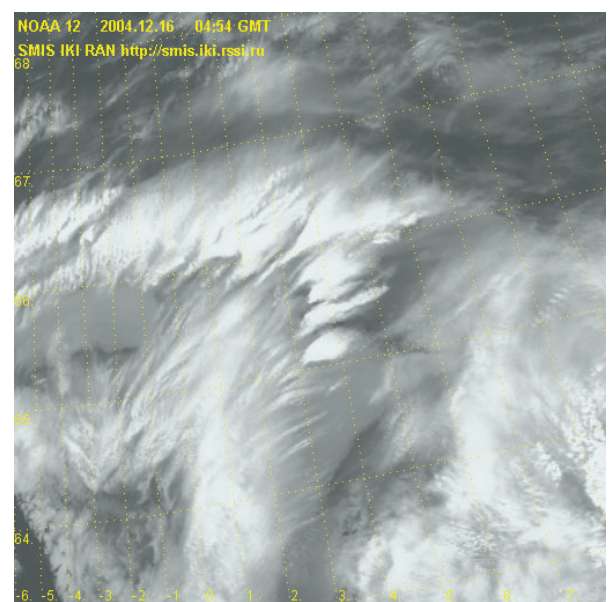

FIGURE 5: The fragment of regional cloud cover distribution data over the part of Norwegian Sea, derived from the results of satellite radiometer monitoring of the Earth's atmosphere, obtained on December 16, 2004 at 04:54 GMT. Geographical coordinates are depicted on the horizontal and vertical axes.

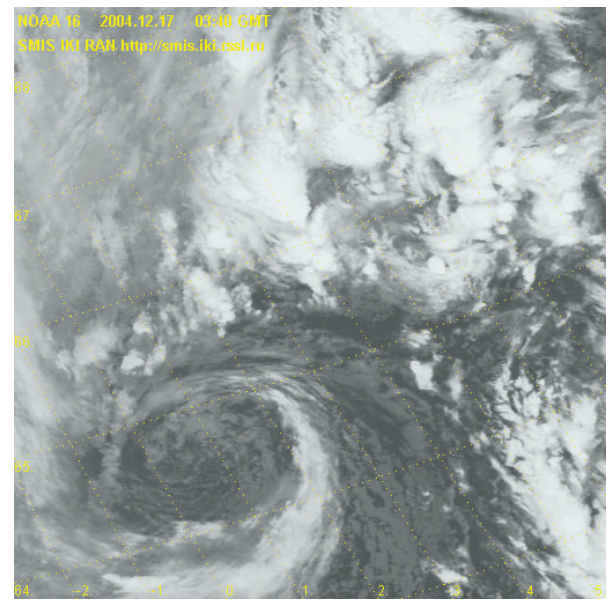

FIgURE 6: The fragment of regional cloud cover distribution data over the part of Norwegian Sea, derived from the results of satellite radiometer monitoring of the Earth's atmosphere, obtained on December 17, 2004 at 03:40 GMT. Geographical coordinates are depicted on the horizontal and vertical axes.

approximately $65^{\circ} \mathrm{N}$ and longitude of approximately $1^{\circ} \mathrm{E}$ was formed. These data correspond qualitatively to the simulation results, obtained in the present subsection.

\section{Summary and Concluding Remarks}

In a number of earlier works, the mechanisms of formation of polar lows have been discussed (e.g., see [10-22]). It should be emphasized that, in earlier studies, we have not found the idea that the transformation of the configuration of the arctic front can influence the process of the formation of polar lows (with the exception of the studies by the authors of the present work), although it was known that the shape of the arctic front may be different. It is evident that the north south position of the arctic front responds to changes in temperature contrast of air masses situated at arctic altitudes and at lower altitudes. It may be recalled that the arctic front separates the cold arctic air masses from warmer air masses.

In the present study, the influence of the disturbance of the configuration of the arctic front on the process of the initial formation of polar lows was investigated numerically. To investigate this process the limited-area nonhydrostatic mathematical model of the wind system of the lower atmosphere, developed recently in the Polar Geophysical Institute, was utilized. The model produces three-dimensional distributions of the atmospheric parameters in the height range from land and ocean surface up to the altitude of $15 \mathrm{~km}$ over a limited region of the Earth's surface. The time evolution of model parameters was numerically simulated using various variants of the initial and boundary conditions which were defined as consistent with the situation when the arctic front intersects the simulation domain in the west-east direction. Calculations were made for various cases in which the initial forms of the arctic front were different and contained convexities with distinct shapes, with the distributions of wind velocity in the vicinity of the arctic front being chosen different.

The simulation results indicated that the origin of a convexity in the configuration of the arctic front, having the latitudinal dimension of about $600 \mathrm{~km}$ and the deviation of hundred kilometers either in north or south direction, can lead to the formation of polar lows during the period of about one day.

The simulation results indicated that, at the presence at the initial moment of the convexity in the configuration of the arctic front in the south direction, eastward from this convexity, approximately in $20 \mathrm{~h}$, a cyclonic vortex is formed which moves southward or in the south-east direction with a velocity of approximately $10-11 \mathrm{~km} / \mathrm{h}$. The maximum wind velocity within this vortex reaches $17.5 \mathrm{~m} / \mathrm{s}$ and the horizontal dimension across this vortex is approximately $600-800 \mathrm{~km}$, with its center being primarily close to the southern edge of the initial arctic front. The maximum wind velocity within the vortex is reached approximately $20 \mathrm{~h}$ after the simulation beginning, and then it begins to decrease slowly.

The simulation results indicated that, at the presence at the initial moment of the convexity in the configuration of the arctic front in the north direction, westward from this convexity, approximately in $20 \mathrm{~h}$, a cyclonic vortex is formed which moves southward or in the south-west direction with a velocity of approximately $10-15 \mathrm{~km} / \mathrm{h}$. The maximum wind velocity within this vortex reaches $18 \mathrm{~m} / \mathrm{s}$ and the horizontal dimension across this vortex is approximately $600-800 \mathrm{~km}$, with its center being primarily close to the southern edge of the initial arctic front. The maximum wind velocity within the vortex is reached approximately $20 \mathrm{~h}$ after the simulation beginning, and then it begins to decrease slowly.

The results of modeling show that a key factor in the modeled formation of polar lows is the origin of a convexity in the configuration of the arctic front. As a consequence, instability of the shear air flow arises. This instability leads to considerable transformation of the wind field. As a result, 
the arctic front may be broken and a polar low can be formed in the vicinity of the initial position of the arctic front in the course of time.

It may be noted that the limited-area nonhydrostatic mathematical model of the wind system of the lower atmosphere, utilized in the present study, possesses one specific limitation. Namely, the calculations of the time evolution of the polar lows, arisen in the present study, were limited by the time intervals not longer than two days. Unfortunately, more prolonged time intervals are impossible for the utilized mathematical model because of limited sizes of its simulation domain and owing to tendency of the modeled polar lows to move and to abandon the simulation domain in the course of time.

It may be expected that the simulation results of the present study will be useful for polar low forecasting. The origin of a convexity in the configuration of the arctic front, which may be observed with the help of satellite monitoring of the Earth's atmosphere, is a precursor of the formation of a polar low. Incidentally, depending on the direction of the convexity in the configuration of the arctic front (to the north or to the south) one can predict the region of polar low appearance.

It can be noticed that, in spite of extensive studies dealing with accumulated data of observations and numerical modeling, there were no firmly established physical mechanisms of formation of polar lows. The establishment of such mechanisms will be significant contributions to our knowledge of polar climate. Also, a topical problem consists in constructing an effective method of early prediction of polar low origination, based on the analysis of satellite observational data. It is hoped that the results of the present study may be applied to solving of this problem.

\section{Conflict of Interests}

The authors declare that there is no conflict of interests regarding the publication of this paper.

\section{Acknowledgment}

This work was partly supported by Grant no. 13-01-00063 from the Russian Foundation for Basic Research.

\section{References}

[1] M. W. Douglas, L. S. Fedor, and M. A. Shapiro, "Polar low structure over the northern Gulf of Alaska based on research aircraft observations," Monthly Weather Review, vol. 119, no. 1, pp. 3254, 1991.

[2] M. T. Montgomery and B. F. Farrell, "Polar low dynamics," Journal of the Atmospheric Sciences, vol. 49, no. 24, pp. 2484-2505, 1992.

[3] T. E. Nordeng and E. A. Rasmussen, "A most beautiful polar low. A case study of a polar low development in the Bear Island region," Tellus A, vol. 44, no. 2, pp. 81-99, 1992.

[4] M. W. Douglas, M. A. Shapiro, L. S. Fedor, and L. Saukkonen, "Research aircraft observations of a polar low at the east greenland ice edge," Monthly Weather Review, vol. 123, no. 1, pp. 5-12, 1995.

[5] X. Zhang, J. E. Walsh, J. Zhang, U. S. Bhatt, and M. Ikeda, "Climatology and interannual variability of arctic cyclone activity: 1948 2002," Journal of Climate, vol. 17, no. 12, pp. 2300-2317, 2004.

[6] H. Sodemann, M. Pommier, S. R. Arnold et al., "Episodes of cross-polar transport in the Arctic troposphere during July 2008 as seen from models, satellite, and aircraft observations," Atmospheric Chemistry and Physics Discussions, vol. 10, no. 11, pp. 26361-26410, 2010.

[7] P. Uotila, T. Vihma, A. B. Pezza, I. Simmonds, K. Keay, and A. H. Lynch, "Relationships between Antarctic cyclones and surface conditions as derived from high-resolution numerical weather prediction data," Journal of Geophysical Research D, vol. 116, no. 7, Article ID D07109, 2011.

[8] H. L. Tanaka, A. Yamagami, and S. Takahashi, "The structure and behavior of the arctic cyclone in summer analyzed by the JRA-25/JCDAS data," Polar Science, 2012.

[9] I. Simmonds and I. Rudeva, "The great Arctic cyclone of August 2012," Geophysical Research Letters, vol. 39, no. 23, 2012.

[10] T. W. Harrold and K. A. Browning, "The polar low as a baroclinic disturbance," Quarterly Journal of the Royal Meteorological Society, vol. 95, no. 406, pp. 710-723, 1969.

[11] R. J. Reed, "Cyclogenesis in polar air streams," Monthly Weather Review, vol. 107, no. 1, pp. 38-52, 1979.

[12] E. Rasmussen, "The polar low as an extratropical CISK disturbance," Quarterly Journal, Royal Meteorological Society, vol. 105, no. 445 , pp. 531-549, 1979.

[13] W. H. Schubert and J. J. Hack, "Transformed Eliassen balanced vortex model," Journal of the Atmospheric Sciences, vol. 40, no. 6, pp. 1571-1583, 1983.

[14] K. A. Emanuel, "The theory of hurricanes," Annual Review of Fluid Mechanics, vol. 23, no. 1, pp. 179-196, 1991.

[15] E. A. Rasmussen and J. Turner, Polar Lows: Mesoscale Weather Systems in the Polar Regions, Cambridge University Press, Cambridge, UK, 2003.

[16] G. S. Golitsyn, “Tropical cyclones and polar lows: velocity, size, and energy scales, and relation to the $26^{\circ} \mathrm{C}$ cyclone origin criteria," Advances in Atmospheric Sciences, vol. 26, no. 3, pp. 585598, 2009.

[17] N. W. Nielsen, "An early-autumn polar low formation over the Norwegian Sea," Journal of Geophysical Research D, vol. 102, no. 12, pp. 13955-13973, 1997.

[18] C. Claud, G. Heinemann, E. Raustein, and L. McMurdie, "Polar low le Cygne: satellite observations and numerical simulations," Quarterly Journal of the Royal Meteorological Society, vol. 130, no. 598, pp. 1075-1102, 2004.

[19] W. Yanase, G. Fu, H. Niino, and T. Kato, "A polar low over the Japan sea on 21 January 1997. Part II: a Numerical Study," Monthly Weather Review, vol. 132, no. 7, pp. 1552-1563, 2004.

[20] L. Wu and G. W. Petty, "Intercomparison of bulk microphysics schemes in model simulations of polar lows," Monthly Weather Review, vol. 138, no. 6, pp. 2211-2228, 2010.

[21] H. Mcinnes, J. Kristiansen, J. E. Kristjánsson, and H. Schyberg, "The role of horizontal resolution for polar low simulations," Quarterly Journal of the Royal Meteorological Society, vol. 137, no. 660, pp. 1674-1687, 2011.

[22] I. Føre and T. E. Nordeng, "A polar low observed over the Norwegian Sea on 3-4 March 2008: high-resolution numerical experiments," Quarterly Journal of the Royal Meteorological Society, vol. 138, no. 669, pp. 1983-1998, 2012. 
[23] I. V. Mingalev and V. S. Mingalev, "The global circulation model of the lower and middle atmosphere of the Earth with a given temperature distribution," Mathematical Modeling, vol. 17, pp. 24-40, 2005 (Russian).

[24] I. V. Mingalev, V. S. Mingalev, and G. I. Mingaleva, "Numerical simulation of the global distributions of the horizontal and vertical wind in the middle atmosphere using a given neutral gas temperature field," Journal of Atmospheric and Solar-Terrestrial Physics, vol. 69, no. 4-5, pp. 552-568, 2007.

[25] J. M. Picone, A. E. Hedin, D. P. Drob, and A. C. Aikin, "NRLMSISE-00 empirical model of the atmosphere: statistical comparisons and scientific issues," Journal of Geophysical Research A, vol. 107, no. 12, article 1468, 2002.

[26] I. V. Mingalev, O. V. Mingalev, and V. S. Mingalev, "Model simulation of the global circulation in the middle atmosphere for January conditions," Advances in Geosciences, vol. 15, pp. 11-16, 2008.

[27] I. V. Mingalev, V. S. Mingalev, and G. I. Mingaleva, "Numerical simulation of the global neutral wind system of the Earth's middle atmosphere for different seasons," Atmosphere, vol. 3, pp. 213-228, 2012.

[28] I. V. Mingalev and V. S. Mingalev, "Numerical modeling of the influence of solar activity on the global circulation in the Earth's mesosphere and lower thermosphere," International Journal of Geophysics, vol. 2012, Article ID 106035, 15 pages, 2012.

[29] O. M. Belotserkovskii, I. V. Mingalev, V. S. Mingalev, O. V. Mingalev, and A. M. Oparin, "Mechanism of the appearance of a large-scale vortex in the troposphere above a nonuniformly heated surface," Doklady Earth Sciences, vol. 411, no. 8, pp. 12841288, 2006.

[30] O. M. Belotserkovskii, I. V. Mingalev, V. S. Mingalev, O. V. Mingalev, A. M. Oparin, and V. M. Chechetkin, "Formation of large-scale vortices in shear flows of the lower atmosphere of the earth in the region of tropical latitudes," Cosmic Research, vol. 47, no. 6, pp. 466-479, 2009.

[31] I. V. Mingalev, N. M. Astafieva, K. G. Orlov, V. S. Mingalev, O. V. Mingalev, and V. M. Chechetkin, "Possibility of a detection of tropical cyclones and hurricanes formation according to satellite remote sensing," in Actual Problems in Remote Sensing of the Earth From Space, vol. 8, pp. 290-296, Space Research Institute, Moscow, Russia, 2011, (Russian).

[32] I. V. Mingalev, N. M. Astafieva, K. G. Orlov, V. M. Chechetkin, V. S. Mingalev, and O. V. Mingalev, "Numerical simulation of formation of cyclone vortex flows in the intertropical zone of convergence and their early detection," Cosmic Research, vol. 50, no. 3, pp. 233-248, 2012.

[33] I. V. Mingalev, N. M. Astafieva, K. G. Orlov, V. S. Mingalev, O. V. Mingalev, and V. M. Chechetkin, "A simulation study of the formation of large-scale cyclonic and anticyclonic vortices in the vicinity of the intertropical convergence zone," ISRN Geophysics, vol. 2013, Article ID 215362, 12 pages, 2013.

[34] I. V. Mingalev, K. G. Orlov, and V. S. Mingalev, "A mechanism of formation of polar lows and possibility of their prediction," in Actual Problems in Remote Sensing of the Earth From Space, vol. 8, pp. 255-262, Space Research Institute, Moscow, Russia, 2011, (Russian).

[35] I. V. Mingalev, K. G. Orlov, and V. S. Mingalev, "A mechanism of formation of polar cyclones and possibility of their prediction using satellite observations," Cosmic Research, vol. 50, no. 2, pp. 160-169, 2012.
[36] V. S. Mingalev, I. V. Mingalev, O. V. Mingalev, A. M. Oparin, and K. G. Orlov, "Generalization of the hybrid monotone secondorder finite difference scheme for gas dynamics equations to the case of unstructured 3D grid," Computational Mathematics and Mathematical Physics, vol. 50, no. 5, pp. 877-889, 2010. 

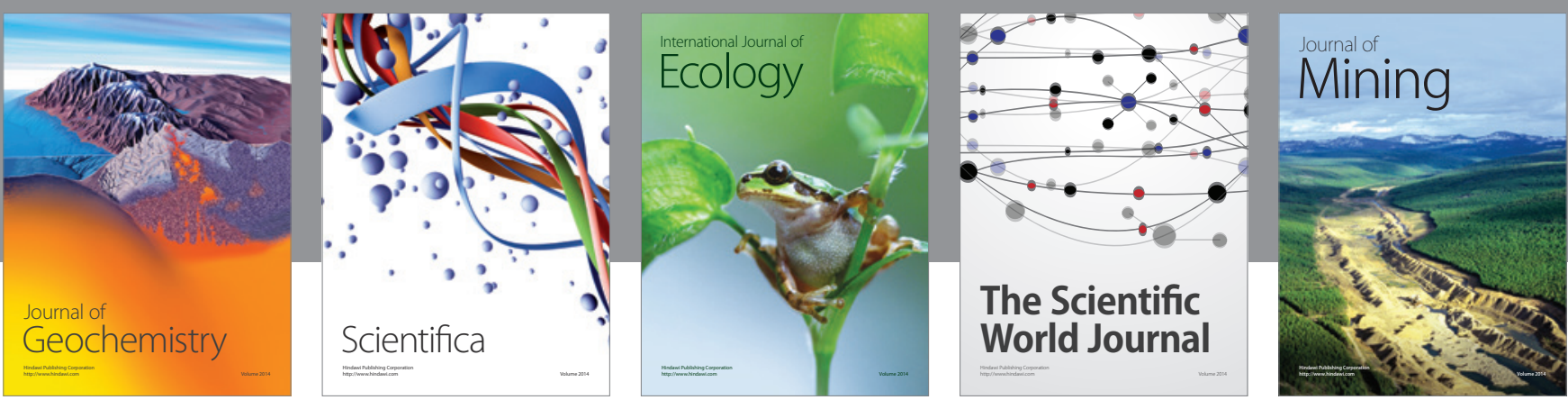

The Scientific World Journal
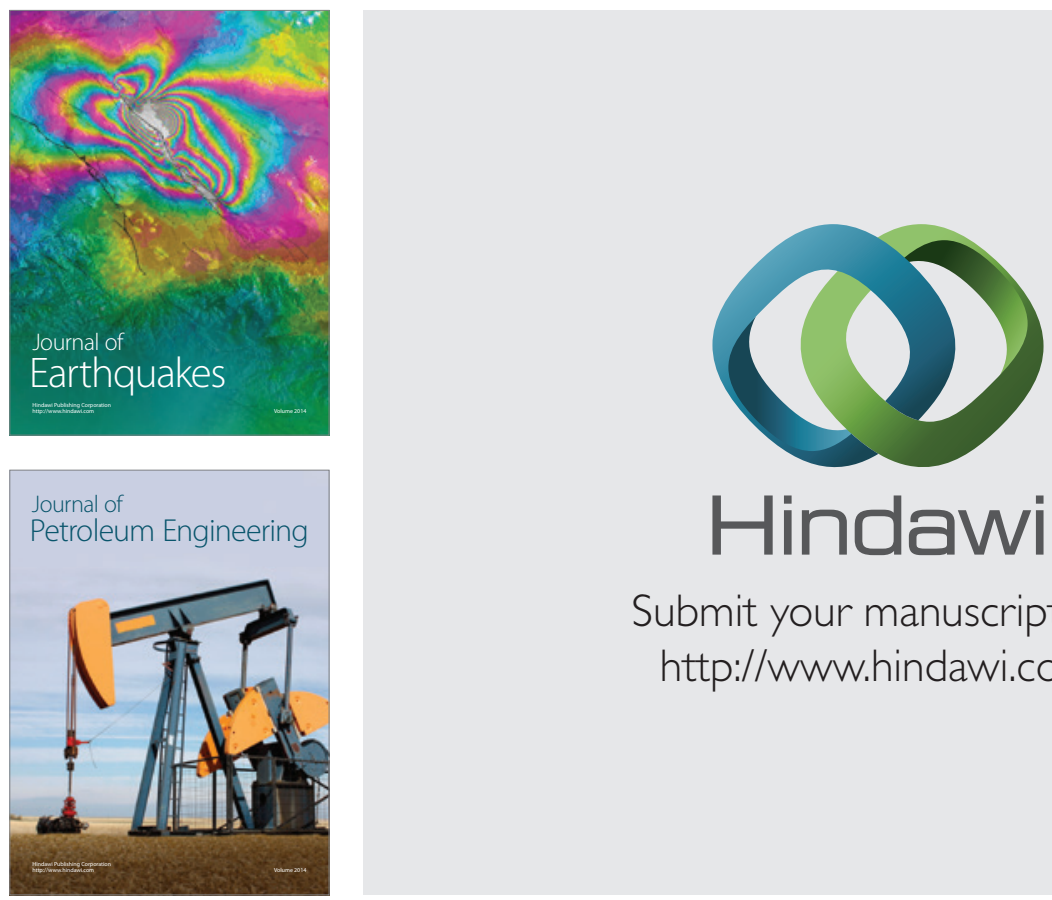

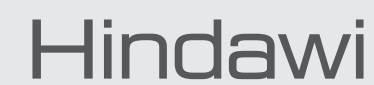

Submit your manuscripts at

http://www.hindawi.com
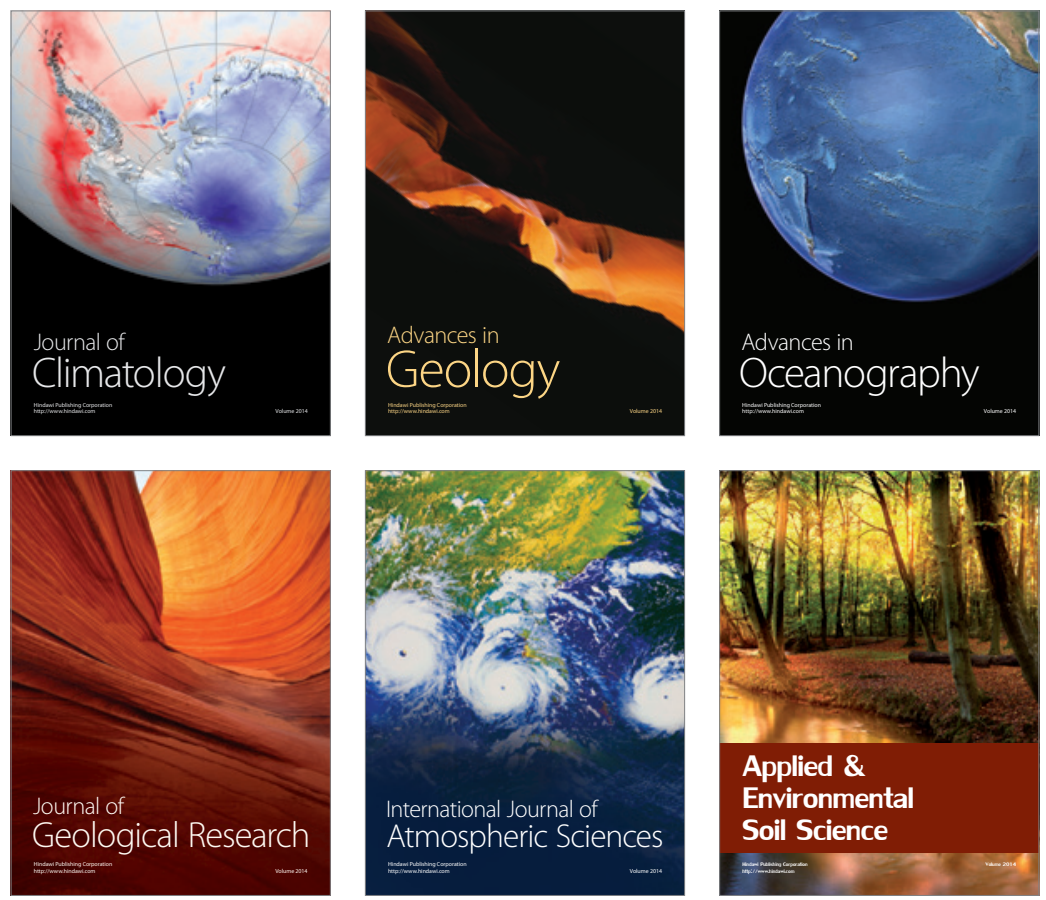
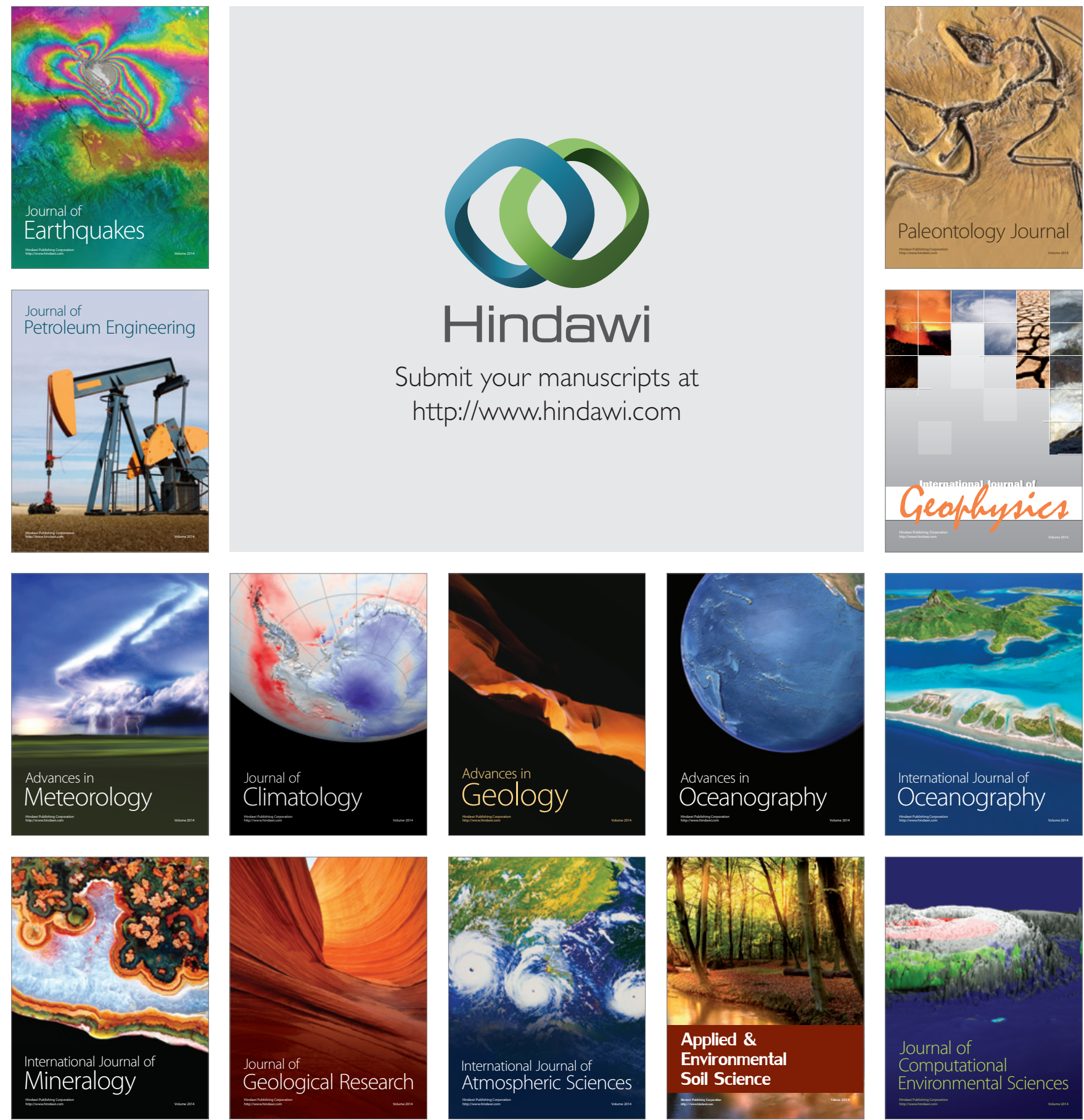